\title{
Buprenorphine for the Treatment of the Neonatal Abstinence Syndrome.
}

\author{
Walter K. Kraft \\ Thomas Jefferson University \\ Susan C. Adeniyi-Jones \\ Thomas Jefferson University \\ Inna Chervoneva \\ Thomas Jefferson University \\ Jay S. Greenspan \\ Thomas Jefferson University \\ Eollow this and additional works at: https://jdc.jefferson.edu/obgynfp
Diane J. Abatemarco \\ JPlatis ofetfferislearldhibgrsitymmons, Obstetrics and Gynecology Commons, and the Pediatrics Commons \\ Let us know how access to this document benefits you
}

See next page for additional authors

\section{Recommended Citation}

Kraft, Walter K.; Adeniyi-Jones, Susan C.; Chervoneva, Inna; Greenspan, Jay S.; Abatemarco, Diane J.; Kaltenbach, Karol; and Ehrlich, Michelle E., "Buprenorphine for the Treatment of the Neonatal Abstinence Syndrome." (2017). Department of Obstetrics and Gynecology Faculty Papers. Paper 42.

https://jdc.jefferson.edu/obgynfp/42

This Article is brought to you for free and open access by the Jefferson Digital Commons. The Jefferson Digital Commons is a service of Thomas Jefferson University's Center for Teaching and Learning (CTL). The Commons is a showcase for Jefferson books and journals, peer-reviewed scholarly publications, unique historical collections from the University archives, and teaching tools. The Jefferson Digital Commons allows researchers and interested readers anywhere in the world to learn about and keep up to date with Jefferson scholarship. This article has been accepted for inclusion in Department of Obstetrics and Gynecology Faculty Papers by an authorized administrator of the Jefferson Digital Commons. For more information, please contact: JeffersonDigitalCommons@jefferson.edu. 
Authors

Walter K. Kraft, Susan C. Adeniyi-Jones, Inna Chervoneva, Jay S. Greenspan, Diane J. Abatemarco, Karol Kaltenbach, and Michelle E. Ehrlich 
ORIGINAL ARTICLE

\section{Buprenorphine for the Treatment of the Neonatal Abstinence Syndrome}

\author{
Walter K. Kraft, M.D., Susan C. Adeniyi-Jones, M.D., Inna Chervoneva, Ph.D., \\ Jay S. Greenspan, M.D., Diane Abatemarco, Ph.D., Karol Kaltenbach, Ph.D., \\ and Michelle E. Ehrlich, M.D.
}

ABSTRACT

\section{BACKGROUND}

Current pharmacologic treatment of the neonatal abstinence syndrome with morphine is associated with a lengthy duration of therapy and hospitalization. Buprenorphine may be more effective than morphine for this indication.

\section{METHODS}

In this single-site, double-blind, double-dummy clinical trial, we randomly assigned 63 term infants ( $\geq 37$ weeks of gestation) who had been exposed to opioids in utero and who had signs of the neonatal abstinence syndrome to receive either sublingual buprenorphine or oral morphine. Infants with symptoms that were not controlled with the maximum dose of opioid were treated with adjunctive phenobarbital. The primary end point was the duration of treatment for symptoms of neonatal opioid withdrawal. Secondary clinical end points were the length of hospital stay, the percentage of infants who required supplemental treatment with phenobarbital, and safety.

\section{RESULTS}

The median duration of treatment was significantly shorter with buprenorphine than with morphine (15 days vs. 28 days), as was the median length of hospital stay ( 21 days vs. 33 days) ( $\mathrm{P}<0.001$ for both comparisons). Adjunctive phenobarbital was administered in 5 of 33 infants (15\%) in the buprenorphine group and in 7 of 30 infants $(23 \%)$ in the morphine group $(\mathrm{P}=0.36)$. Rates of adverse events were similar in the two groups.

\section{CONCLUSIONS}

Among infants with the neonatal abstinence syndrome, treatment with sublingual buprenorphine resulted in a shorter duration of treatment and shorter length of hospital stay than treatment with oral morphine, with similar rates of adverse events. (Funded by the National Institute on Drug Abuse; BBORN ClinicalTrials.gov number, NCT01452789.)
From Sidney Kimmel Medical College, Thomas Jefferson University (W.K.K., S.C.A.-J., I.C., J.S.G., D.A., K.K.), and Nemours duPont Pediatrics, Thomas Jefferson University Hospital (S.C.A.-J., J.S.G.) - both in Philadelphia; and the Departments of Neurology, Pediatrics, and Genetics and Genomic Sciences, Icahn School of Medicine at Mount Sinai, New York (M.E.E.). Address reprint requests to Dr. Kraft at the Department of Pharmacology and Experimental Therapeutics, Sidney Kimmel Medical College, Thomas Jefferson University, 1170 Main Bldg., 132 S. 10th St., Philadelphia, PA 19107-5244, or atwalter.kraft@jefferson.edu.

This article was published on May 4, 2017, at NEJM.org.

DOI: 10.1056/NEJMoa1614835

Copyright $\odot 2017$ Massachusetts Medical Society. 
T HE NEONATAL ABSTINENCE SYNDROME is defined as the occurrence of signs and symptoms of neonatal withdrawal after in utero drug exposure. ${ }^{1}$ Among drug exposures, opioids cause severe symptoms, including autonomic instability, tremor, irritability, poor feeding, and loose stool. Measures that improve symptom control include minimization of stimulation, rooming in, ${ }^{2}$ breast-feeding, ${ }^{3}$ and frequent calorically dense feedings. Approximately two thirds of infants with this condition do not have a response to behavioral approaches and ultimately require pharmacologic therapy for control of symptoms. ${ }^{4}$

The administration of an opioid at an appropriate dose for symptom control with subsequent weaning has been identified as an effective approach, but data are limited to guide the choice of opioid. ${ }^{5}$ Morphine is used in more than $80 \%$ of the infants who require treatment for the neonatal abstinence syndrome in the United States. ${ }^{4}$ In adults, sublingual buprenorphine is used to reduce symptoms of opioid withdrawal. ${ }^{6}$ Buprenorphine has a wide therapeutic index for respiratory depression and a long half-life that may make it useful for treatment of the neonatal abstinence syndrome. Open-label investigations have established a pharmacokinetic profile in neonates ${ }^{7}$ and suggested favorable safety and efficacy for this indication. ${ }^{8,9}$ In the single-site, randomized, double-blind, double-dummy Blinded Buprenorphine or Neonatal Morphine Solution (BBORN) trial, we compared sublingual buprenorphine with oral morphine with respect to the duration of treatment in infants with the neonatal abstinence syndrome.

\section{METHODS}

\section{TRIAL DESIGN AND POPULATION}

From October 31, 2011, to May 29, 2016, we enrolled term infants ( $\geq 37$ weeks of gestation) who had been exposed to opioids in utero and had signs and symptoms of the neonatal abstinence syndrome. Exclusion criteria were a major congenital malformation, a birth weight of less than $2200 \mathrm{~g}$, a serious medical or neurologic illness, hypoglycemia requiring intravenous glucose, a bilirubin level of more than $20 \mathrm{mg}$ per deciliter (342 $\mu \mathrm{mol}$ per liter), maternal use of a benzodiazepine 30 days before birth, or seizures. Breast- feeding was an exclusionary criterion until the approval of a protocol amendment on October 17,2013 . This expansion was driven by changing local and national ${ }^{4}$ practice patterns during the trial, which increased the number of women who attempted to breast-feed. Written informed consent was obtained from the parents.

The trial was approved by the institutional review board at Thomas Jefferson University. The drug manufacturer, Indivior, donated the buprenorphine that was used in the trial but was not involved in the trial design, in the collection, analysis, or interpretation of the data, or in the preparation of the manuscript. The protocol, which includes the statistical analysis plan, is available with the full text of this article at NEJM.org.

\section{TREATMENT}

We monitored all infants for the severity of the neonatal abstinence syndrome using the MOTHER NAS scale, a modified Finnegan scoring instrument,${ }^{10}$ which ranges from 0 to 42 (typical range in practice, approximately 2 to 14), with higher scores indicating greater severity. (Details regarding this scale are provided in Table S1 in the Supplementary Appendix, available at NEJM.org.) Scoring on this scale was performed every 4 hours for a minimum of 72 hours. The threshold for pharmacologic treatment was a sum of three scores of 24 or more or a single score of 12 or more.

Infants for whom treatment was required were randomly assigned in a 1:1 ratio to receive either sublingual buprenorphine or oral morphine and the corresponding placebo. Randomization was stratified according to maternal exposure to methadone or buprenorphine and the maternal intention to breast-feed or bottle-feed.

The dose and regimen of buprenorphine were derived from efficacy, pharmacokinetic, and safety data generated in a phase 1 trial., ${ }^{8,9}$ Buprenorphine was administered at a dose of $0.075 \mathrm{mg}$ per milliliter of solution in simple syrup and 30\% ethanol, which is stable for at least 7 days at room temperature. ${ }^{11}$ Placebo for buprenorphine contained no ethanol $(1.75 \mathrm{ml}$ of simple syrup in $2.25 \mathrm{ml}$ of sterile water). The dose of morphine was based on the standard-of-care protocol at Thomas Jefferson University Hospital (Table S10 in the Supplementary Appendix). Morphine at a dose of $0.4 \mathrm{mg}$ per milliliter was formulated 
with the use of an oral solution of $4 \mathrm{mg}$ per milliliter (Roxane) and dilution with sterile water, which is stable for at least 60 days at room temperature. ${ }^{12}$ Placebo for morphine was sterile water mixed with blue dye.

Buprenorphine or placebo was administered sublingually every 8 hours by a syringe under the tongue. A pacifier was placed in the infant's mouth to maximize contact time with the sublingual mucosa. For doses in which the volume was greater than $0.5 \mathrm{ml}$, buprenorphine or placebo was given in two administrations separated by at least 2 minutes. Morphine or placebo was administered orally every 4 hours.

Doses of buprenorphine or morphine were adjusted according to disease severity. After the first four infants had undergone randomization (three in the buprenorphine group and one in the morphine group), we amended the protocol to change the rate of increase in the morphine dose from $10 \%$ to $20 \%$ to match changes in the hospital standard of care (Fig. S1 in the Supplementary Appendix). After infants had symptom stability for at least 48 hours, doses were decreased by $10 \%$ once per day if the sum of three scores was less than 18 . The investigational-drug pharmacist notified the clinical team when the cessation dose had been reached. Infants were observed in the hospital for at least 48 hours after the last dose of a trial medication was administered. A rescue dose after cessation of therapy could be given in a blinded manner at the discretion of the treating physician in any infant with a score of 12 or more.

The maximum dose was $60 \mu \mathrm{g}$ per kilogram of body weight per day for buprenorphine and $1.2 \mathrm{mg}$ per kilogram per day for morphine (Table S2 in the Supplementary Appendix). The maximum dose in the two groups was reached after six increases in dose, which maintained blinding. If disease stability was not obtained at the maximum opioid dose, phenobarbital was initiated with a loading dose of $20 \mathrm{mg}$ per kilogram, followed by a daily oral dose of $5 \mathrm{mg}$ per kilogram per day. When the opioid was weaned to $50 \%$ of the highest dose, phenobarbital was decreased to $2.5 \mathrm{mg}$ per kilogram per day. After three additional steps in opioid weaning, phenobarbital was discontinued and the opioid was weaned until the cessation dose was reached. Liver-function testing was performed before or soon after the initiation of a trial drug and at 7 and 21 days after the initiation of therapy.

\section{END POINTS}

The primary end point was the duration of treatment for neonatal withdrawal symptoms in days from the first dose of a trial drug. Secondary clinical end points were the length of hospital stay (including all levels of care), the percentage of infants who required supplemental treatment with phenobarbital, and safety. Exploratory end points included respiratory rate and weight gain. Other end points that are not discussed here included pharmacokinetic measurements of buprenorphine and morphine (secondary outcomes) and pharmacogenetic, neurobehavioral, and feedingdysfunction measures (exploratory outcomes).

\section{STATISTICAL ANALYSIS}

The primary analysis was performed according to the intention-to-treat principle. On the basis of the results of previous open-label trials, we determined that a sample size of 40 infants in each trial group would provide a power of $90 \%$ to detect a $28 \%$ shorter duration of treatment in the buprenorphine group than in the morphine group (a difference of 0.323 on a log scale), assuming a common standard deviation of 0.44 . Owing to slower-than-anticipated patient recruitment, the data and safety monitoring board agreed to a suggestion from the investigators to stop enrollment before the recruitment of 80 infants.

The randomization was stratified according to the mother's intended feeding pattern (bottlefeeding vs. breast-feeding) and maternal use of buprenorphine versus methadone. However, only 3 infants whose mothers were receiving buprenorphine underwent randomization (1 in the buprenorphine group and 2 in the morphine group) and all were bottle-fed, so stratification of the analysis according to maternal use of buprenorphine was not meaningful. Therefore, statistical tests were adjusted only for the type of feeding. Neither the duration of treatment nor the log-transformed duration of treatment was consistent with normal distribution assumptions. Therefore, a stratified two-sample van Elteren test $^{13}$ (an extension of the Wilcoxon rank-sum test) was used to compare the duration of treatment and the length of hospital stay in the two 


\begin{tabular}{|c|c|c|}
\hline Characteristic & $\begin{array}{l}\text { Buprenorphine } \\
\qquad(\mathrm{N}=33)\end{array}$ & $\begin{array}{l}\text { Morphine } \\
(\mathrm{N}=30)\end{array}$ \\
\hline \multicolumn{3}{|l|}{ Infants } \\
\hline Median gestational age (range) $-w k$ & $38.5(37.0-42.0)$ & $39.0(37.0-41.0)$ \\
\hline Median birth weight (range) - $\mathrm{g}$ & $3040(2270-4380)$ & $3004(2197-3850)$ \\
\hline Male sex - no. (\%) & $17(52)$ & $18(60)$ \\
\hline Breast-feeding subgroup — no. (\%) $\dagger$ & $12(36)$ & $9(30)$ \\
\hline \multicolumn{3}{|l|}{ Median Apgar score (range) } \\
\hline 1 & $8(3-9)$ & $8(5-10)$ \\
\hline 2 & $9(7-9)$ & $9(7-10)$ \\
\hline \multicolumn{3}{|l|}{ Race - no. $(\%) \ddagger$} \\
\hline White & $28(85)$ & $24(80)$ \\
\hline Black & $5(15)$ & $4(13)$ \\
\hline Other & 0 & $2(7)$ \\
\hline Median head circumference (range) $-\mathrm{cm}$ & $33.0(30.0-36.5)$ & $33.0(27.5-36.5)$ \\
\hline Age at treatment initiation (range) - days & $2(1-9)$ & $2(1-14)$ \\
\hline \multicolumn{3}{|l|}{ Mothers } \\
\hline \multicolumn{3}{|l|}{ Use of methadone } \\
\hline Maintenance therapy — no. (\%) & $32(97)$ & $27(90)$ \\
\hline Daily dose (range) - mg & $130(25-265)$ & $135(30-260)$ \\
\hline \multicolumn{3}{|l|}{ Use of buprenorphine } \\
\hline Maintenance therapy — no. (\%) & $1(3)$ & $2(7)$ \\
\hline Daily dose (range) - mg & 8 & $7(6-8)$ \\
\hline Use of short-acting opioid — no. (\%) & 0 & $1(3)$ \\
\hline \multicolumn{3}{|l|}{ Use of tobacco - no. (\%) } \\
\hline Any & $28(85)$ & $28(93)$ \\
\hline More than 5 cigarettes per day & $22(67)$ & $22(73)$ \\
\hline \multicolumn{3}{|l|}{ Drugs identified on urine screening - no. (\%) } \\
\hline Cocaine & $1(3)$ & $5(17)$ \\
\hline Amphetamine & $1(3)$ & 0 \\
\hline Other opioid & $3(9)$ & $5(17)$ \\
\hline
\end{tabular}

* There were no significant differences between the groups except for median gestational age $(P=0.03)$.

$\uparrow$ Stratification according to breast-feeding or bottle-feeding was determined by the mother's intent at the time of randomization; actual behavior after randomization was variable.

$\uparrow$ Race was reported by the mothers.

groups after adjustment for differences in the type of feeding. A Cochran-Mantel-Haenszel test was used to evaluate the association between trial group and the use of supplemental phenobarbital, stratified according to the type of feeding.

No interim analysis was performed. For the exploratory end points of respiratory rate and weight gain and post hoc safety assessment of heart rate before and during treatment, longitudinal data were modeled in a linear mixed-effects model with the time trends represented by loworder polynomials in postnatal days, with the polynomial coefficients dependent on fixed effects of drug and feeding type and random effects of between-patient variation. When appropriate, the variance components were estimated separately 


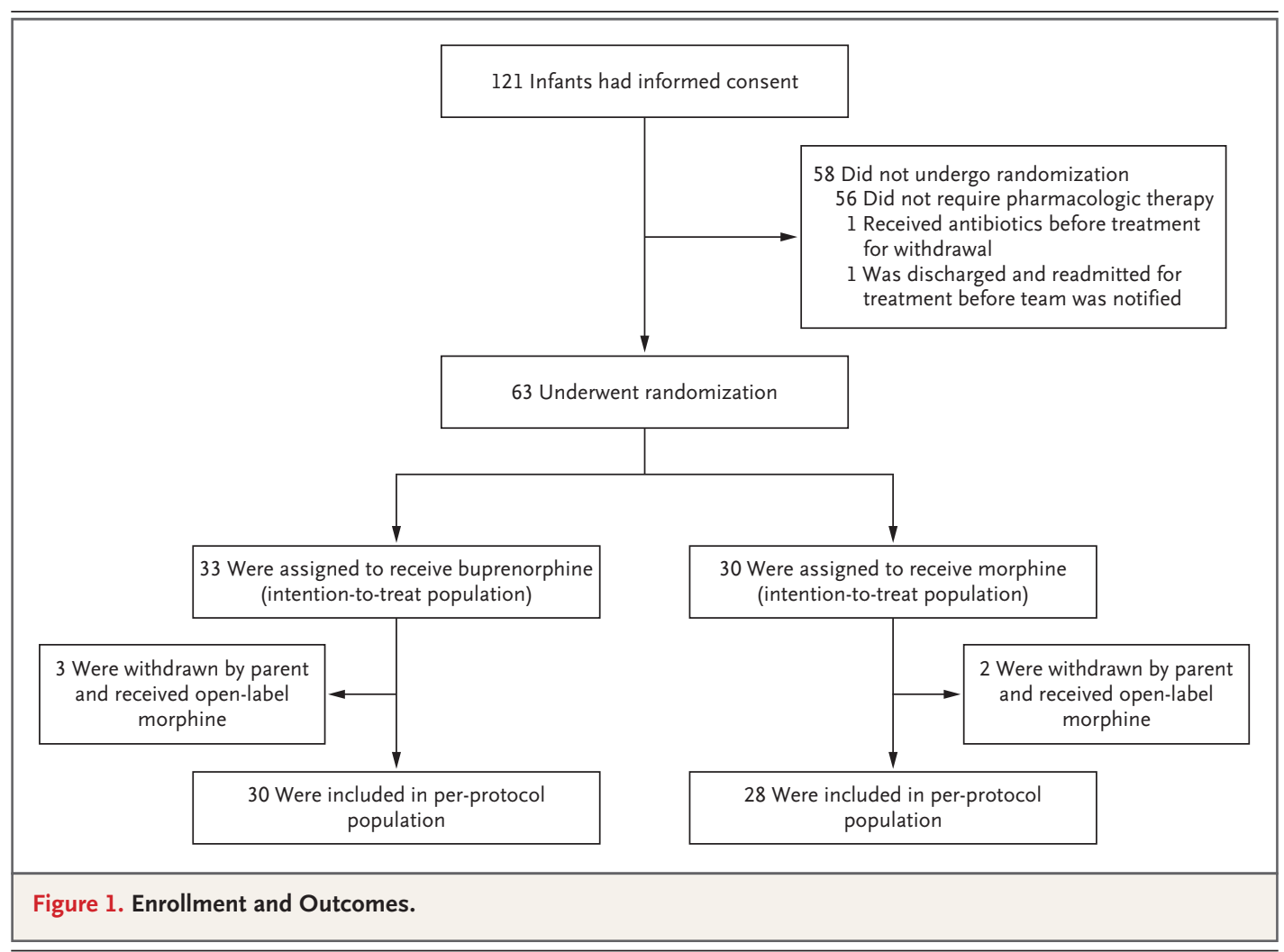

for each group. The degrees of freedom were computed with the use of the approach of Kenward and Roger. ${ }^{14}$ The tests were not adjusted for multiplicity because of their exploratory nature. All tests were two-sided. Statistical analyses were performed with the use of SAS software, version 9.4 (SAS Institute), and R software (R Foundation for Statistical Computing).

\section{RESULTS}

\section{PATIENTS}

Of the 121 singleton infants who underwent screening, 63 were eligible for pharmacologic therapy and were enrolled (Table 1 and Fig. 1). Mothers were predominantly enrolled in an outpatient methadone treatment program. On the basis of maternal self-declaration of feeding plans, 21 infants were assigned to the breast-feeding subgroup (12 in the buprenorphine group and 9 in the morphine group). The median duration of breast-feeding while receiving a trial drug was 7 days (range, 0 to 42) during a median duration of treatment of 23 days. At the time of hospital discharge, 9 of 21 infants (43\%) were breast-fed.
Six infants (29\%) were breast-fed for no more than 2 days before their mothers decided to switch exclusively to bottle feeding.

After randomization, the parents of 5 infants withdrew consent for participation in the trial. In 4 infants ( 2 in the buprenorphine group and 2 in the morphine group), the parental concern was based on the slow duration of the weaning process. In the buprenorphine group, a parent decided to withdraw 1 infant after two doses of the trial drug were administered. No withdrawals were due to adverse events. After withdrawal from the trial, all the infants were treated with openlabel morphine according to the same treatment protocol.

In the intention-to-treat analysis, 33 infants were included in the buprenorphine group and 30 infants in the morphine group. In the perprotocol analysis, 5 infants who had undergone randomization were excluded from the analysis because of withdrawal of consent. Thus, the perprotocol analysis included 30 infants in the buprenorphine group and 28 infants in the morphine group. In the as-treated analysis, 3 infants who had been assigned to the buprenorphine 


\begin{tabular}{|c|c|c|c|c|}
\hline Outcome and Feeding Subgroup & $\begin{array}{l}\text { Buprenorphine } \\
\qquad(\mathrm{N}=33)\end{array}$ & $\begin{array}{c}\text { Morphine } \\
(\mathrm{N}=30)\end{array}$ & $\begin{array}{c}\text { Difference } \\
(95 \% \mathrm{CI}) * \\
\text { days }\end{array}$ & P Value \\
\hline \multicolumn{5}{|l|}{ No. of infants } \\
\hline Bottle-feeding & 21 & 21 & & \\
\hline Breast-feeding & 12 & 9 & & \\
\hline \multicolumn{5}{|l|}{ Primary outcome } \\
\hline Median duration of treatment (range) - days & 15 (3 to 67$)$ & 28 (13 to 67$)$ & $-13(-21$ to -7$)$ & $<0.001 \dagger$ \\
\hline Bottle-feeding & 15 (3 to 67$)$ & 28 (13 to 67 ) & $-13(-23$ to -6$)$ & \\
\hline Breast-feeding & 20 (3 to 55$)$ & 28 (16 to 52 ) & $-8(-30$ to 2$)$ & \\
\hline \multicolumn{5}{|l|}{ Secondary outcomes } \\
\hline Median length of hospital stay (range) - days & 21 (7 to 71$)$ & 33 (18 to 70$)$ & $-12(-22$ to -7$)$ & $<0.001 \dagger$ \\
\hline Bottle-feeding & 21 (7 to 71$)$ & 33 (18 to 70$)$ & $-12(-23$ to -7$)$ & \\
\hline Breast-feeding & 26 (7 to 58$)$ & 32 (20 to 58$)$ & $-8(-29$ to 2$)$ & \\
\hline Use of supplemental phenobarbital — no. (\%) & $5(15)$ & $7(23)$ & & $0.36+$ \\
\hline Bottle-feeding & $2(6)$ & $4(13)$ & & \\
\hline Breast-feeding & $3(9)$ & $3(10)$ & & \\
\hline
\end{tabular}

* The difference between the buprenorphine group and the morphine group was calculated with the use of the Hodges-Lehmann estimator as the median of all paired differences between observations in the two groups with the corresponding nonparametric $95 \%$ confidence interval $(\mathrm{Cl})$.

$\dagger$ This $\mathrm{P}$ value was calculated by means of the van Elteren test.

$\checkmark$ This $P$ value was calculated by means of the Cochran-Mantel-Haenszel test.

group but had withdrawal of consent were included in the morphine group. Thus, 30 infants were included in the buprenorphine group and 33 infants in the morphine group in the astreated analysis.

\section{PRIMARY AND SECONDARY END POINTS}

In the intention-to-treat analysis, the median duration of treatment was 15 days in the buprenorphine group and 28 days in the morphine group, a difference of 13 days $(95 \%$ confidence interval [CI], 7 to 21; $\mathrm{P}<0.001)$. The median length of hospital stay was 21 days in the buprenorphine group and 33 days in the morphine group, a difference of 12 days (95\% CI, 7 to 22; $\mathrm{P}<0.001$ ) (Table 2 and Fig. 2). The duration of treatment and length of hospital stay were also significantly shorter in the buprenorphine group than in the morphine group in both the per-protocol analysis and the as-treated analysis $(\mathrm{P}<0.001$ for both comparisons) (Tables S3 and S4 in the Supplementary Appendix).

The use of supplemental phenobarbital was required in 5 of 33 infants (15\%) in the bu- prenorphine group ( 2 in the bottle-feeding subgroup and 3 in the breast-feeding subgroup) and in 7 of 30 infants (23\%) in the morphine group (4 in the bottle-feeding subgroup and 3 in the breast-feeding subgroup) $(\mathrm{P}=0.36)$. No infants were readmitted to the hospital after discharge. In a sensitivity analysis, the primary finding was materially unchanged when the 3 infants who were assigned to the buprenorphine group but subsequently received morphine were hypothetically assumed to have a duration of treatment equal to the maximum of all observed values instead of actually observed values $(\mathrm{P}<0.001)$.

\section{SAFETY}

Overall, 13 adverse events occurred in 7 infants in the buprenorphine group and 10 events in 8 infants in the morphine group ( $\mathrm{P}=0.79$ for the number of events by Fisher's exact test) (Table 3). There were two serious adverse events. One was an inguinal hernia repair in the morphine group and one was a supraglottoplasty associated with the Pierre Robin syndrome in the buprenorphine group. There were no eleva- 
tions in levels of alanine aminotransferase or aspartate aminotransferase in any infant. The mean respiratory rate in the morphine group was lower by 4.4 breaths per minute ( $95 \%$ CI, 0.7 to 8.1) than in the buprenorphine group in the same feeding subgroup $(\mathrm{P}=0.02)$, although all the respiratory rates were within the normal range. At 7 days, the mean reduction in weight from birth was less in the morphine group than in the buprenorphine group (167 g vs. $231 \mathrm{~g}$, $\mathrm{P}=0.04$ ). There were no significant betweengroup differences in the change from birth weight or in heart rates at days 14, 21, and 28.

\section{DISCUSSION}

In this single-center, randomized trial involving 63 infants with the neonatal abstinence syndrome, we found that buprenorphine was significantly more effective than morphine in reducing the duration of treatment, with a median between-group difference of 13 days in the intention-to-treat analysis. This difference translated into a similarly significant reduction in the length of hospital stay. Results were similar in the per-protocol analysis and the as-treated analysis. We found no significant differences between groups in the need for adjunctive phenobarbital treatment, although few infants in either group received this treatment. These findings suggest that the pharmacodynamic effects of buprenorphine and morphine may not differ substantially at the predefined maximal doses.

The frequency of adverse events was similar in the two groups. Infants in the morphine group had a lower respiratory rate than those in the buprenorphine group. This potential advantage, along with a longer interval between doses, may allow for investigation of buprenorphine in outpatient settings, a use that was not examined in this trial.

Limitations of the trial include the small sample size and the single-center design. Also, since we excluded preterm infants and those with in utero exposure to benzodiazepines, our results should not be generalized to infants with such characteristics. Buprenorphine has been used in critically ill preterm infants, ${ }^{15}$ but its utility in preterm infants with the neonatal abstinence syndrome has not been defined. Preterm infants have a decreased incidence and severity

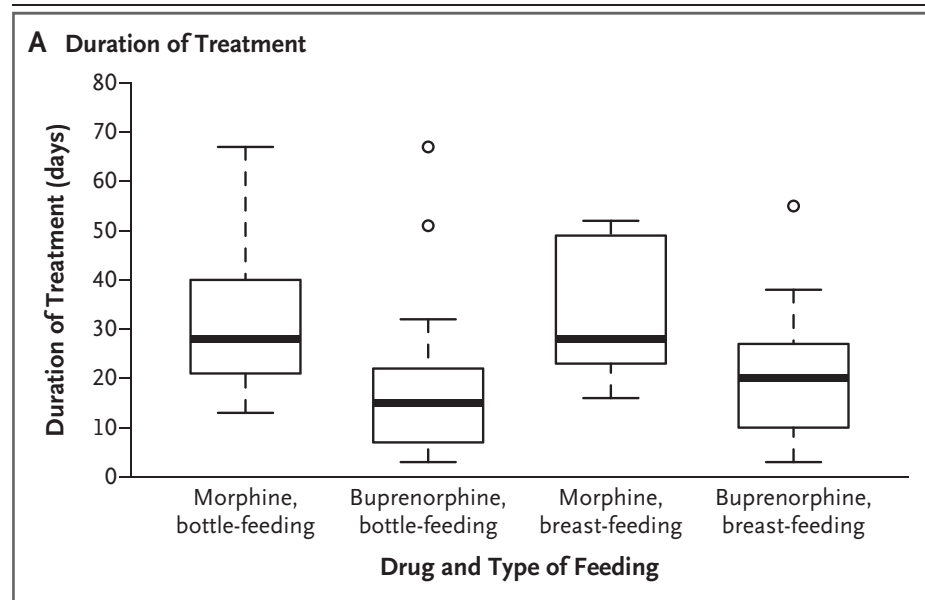

B Length of Hospital Stay

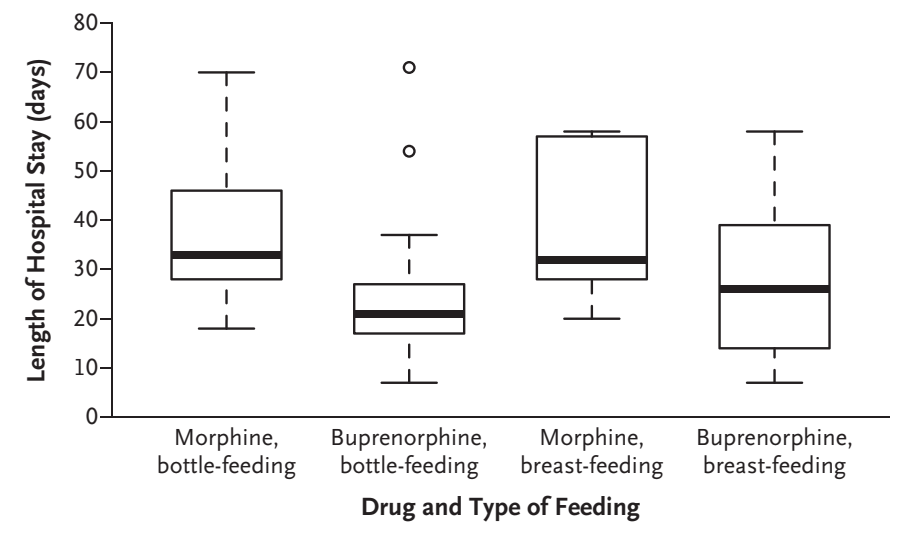

Figure 2. Duration of Treatment and Length of Hospital Stay.

The duration of treatment (Panel A) and length of hospital stay (Panel B) were shorter in the buprenorphine group than in the morphine group $(\mathrm{P}<0.001$ for both comparisons). Randomization was stratified according to the type of intended feeding method (bottle-feeding or breast-feeding). The box-and-whisker plots represent medians (horizontal lines) and interquartile ranges (top and bottom of the boxes); the I bars represent the maximum or minimum value or 1.5 times the interquartile range. Outliers are indicated by circles.

of withdrawal signs, ${ }^{16-18}$ and it is unclear whether the standard scoring instruments that we used would be valid in this population. Exposure to benzodiazepines in utero is associated with worsened symptoms of neonatal abstinence. The use of buprenorphine in infants with benzodiazepine exposure is currently being investigated in other clinical trials (ClinicalTrials.gov numbers, NCT02249026 and NCT01671410).

The mechanisms by which buprenorphine allows for a shorter duration of treatment than morphine are unclear. It is possible that the long 


\begin{tabular}{|lcc|}
\hline Table 3. Adverse Events. & $\begin{array}{c}\text { Buprenorphine } \\
(\mathbf{N}=33)\end{array}$ & $\begin{array}{c}\text { Morphine } \\
(\mathbf{N}=30)\end{array}$ \\
Event & no. of events & \\
Serious adverse events & 0 & 1 \\
Inguinal hernia repair & 1 & 0 \\
Supraglottoplasty & & \\
Other adverse events & 13 & 10 \\
Any & 1 & 0 \\
Anemia & 5 & 3 \\
Skin condition & 3 & 3 \\
Gastrointestinal condition & 0 & 2 \\
Respiratory infection & 1 & 0 \\
Cough & 1 & 0 \\
Tachycardia & 1 & 0 \\
Umbilical granuloma & 0 & 1 \\
Urinary tract infection & & \\
\hline
\end{tabular}

half-life of the drug smooths out peaks and troughs that are seen with morphine and also allows for a more gradual loss of agonism at the mu opioid receptor than that with a short-acting agent. Unlike methadone, another long-acting opioid, buprenorphine is a partial agonist. This functional antagonist effect may also ease the cessation. Agonist effects of buprenorphine at the mu, delta, and opioid receptor-like (ORL1) receptors and antagonism at the kappa receptor may also play a role.

In conclusion, we found that sublingually administered buprenorphine was significantly more effective than oral morphine in reducing the duration of treatment for the neonatal abstinence syndrome, which translated into a shorter hospital stay. The two drugs had similar safety profiles.

Supported by a grant (R01DA02976) from the National Institute on Drug Abuse.

Disclosure forms provided by the authors are available with the full text of this article at NEJM.org.

\section{REFERENCES}

1. Hudak ML, Tan RC. Neonatal drug withdrawal. Pediatrics 2012;129(2):e540e560.

lingual buprenorphine in neonatal abstinence syndrome. Pharmacotherapy 2015; 35:670-80.

2. Holmes AV, Atwood EC, Whalen B, et al. Rooming-in to treat neonatal abstinence syndrome: improved family-centered care at lower cost. Pediatrics 2016;137(6): e20152929.

3. Short VL, Gannon M, Abatemarco DJ. The association between breastfeeding and length of hospital stay among infants diagnosed with neonatal abstinence syndrome: a population-based study of inhospital births. Breastfeed Med 2016;11: 343-9.

4. Patrick SW, Schumacher RE, Horbar JD, et al. Improving care for neonatal abstinence syndrome. Pediatrics 2016;137(5): e20153835.

5. Osborn DA, Jeffery HE, Cole MJ. Opiate treatment for opiate withdrawal in newborn infants. Cochrane Database Syst Rev 2010;CD002059.

6. Gowing L, Ali R, White JM, Mbewe D. Buprenorphine for managing opioid withdrawal. Cochrane Database Syst Rev 2017; 2:CD002025.

7. $\mathrm{Ng} \mathrm{CM}$, Dombrowsky E, Lin H, et al. Population pharmacokinetic model of sub-
8. Kraft WK, Gibson E, Dysart K, et al. Sublingual buprenorphine for treatment of neonatal abstinence syndrome: a randomized trial. Pediatrics 2008;122(3): e601-e607.

9. Kraft WK, Dysart K, Greenspan JS Gibson E, Kaltenbach K, Ehrlich ME. Revised dose schema of sublingual buprenoropioid abstinence syndrome. Addiction 2011;106:574-80.

10. Kaltenbach K, Jones HE. Neonatal abstinence syndrome: presentation and treatment considerations. J Addict Med 2016; 10:217-23.

11. Anagnostis EA, Sadaka RE, Sailor LA, Moody DE, Dysart KC, Kraft WK. Formulation of buprenorphine for sublingual use in neonates. J Pediatr Pharmacol Ther 2011;16:281-4

12. Sauberan J, Rossi S, Kim JH. Stability of dilute oral morphine solution for neonatal abstinence syndrome. J Addict Med 2013;7:113-5.

13. Van Elteren PH. On the combination of independent two-sample tests of phine in the treatment of the neonatal
Wilcoxon. Bull Int Stat Inst 1960;37:35161.

14. Kenward MG, Roger JH. Small sample inference for fixed effects from restricted maximum likelihood. Biometrics 1997;53: 983-97.

15. Barrett DA, Simpson J, Rutter N, Kurihara-Bergstrom T, Shaw PN, Davis SS. The pharmacokinetics and physiological effects of buprenorphine infusion in premature neonates. Br J Clin Pharmacol 1993;36:215-9.

16. Dysart K, Hsieh HC, Kaltenbach K, Greenspan JS. Sequela of preterm versus term infants born to mothers on a methadone maintenance program: differential course of neonatal abstinence syndrome. J Perinat Med 2007;35:344-6.

17. Seligman NS, Salva N, Hayes EJ, Dysart KC, Pequignot EC, Baxter JK. Predicting length of treatment for neonatal $\mathrm{ab}$ stinence syndrome in methadone-exposed neonates. Am J Obstet Gynecol 2008; 199(4):396.e1-7.

18. Doberczak TM, Kandall SR, Wilets I. Neonatal opiate abstinence syndrome in term and preterm infants. J Pediatr 1991; 118:933-7.

Copyright (c) 2017 Massachusetts Medical Society. 